\title{
A clinical and basic study of optimal endometrial preparation protocols for patients with infertility undergoing frozen-thawed embryo transfer
}

\author{
JUNXIA AN ${ }^{1,2}$, LIFEI LI ${ }^{1,2}$, XIANG ZHANG ${ }^{1,2}$, LIN LIU $^{1,2}$, LIYAN WANG $^{1,2}$ and XUEHONG ZHANG ${ }^{1,2}$ \\ ${ }^{1}$ The Reproductive Medicine Special Hospital of The First Hospital of Lanzhou University; ${ }^{2}$ Key Laboratory for Reproductive \\ Medicine and Embryo of Gansu, The First Hospital of Lanzhou University, Lanzhou, Gansu 730000, P.R. China
}

Received October 24, 2019; Accepted April 17, 2020

DOI: $10.3892 /$ etm. 2020.8914

\begin{abstract}
The optimal protocol for endometrial preparation in patients with infertility remains unclear. Due to this, the current study retrospectively analyzed 1,589 patients with infertility and regular menstrual cycles to assess reproductive outcomes per embryo transferred and per embryo transfer (ET) cycle following the transfer of frozen-thawed embryos (FET) in a modified natural cycle (mNC) or hormone therapy cycle (HT) with or without gonadotropin-releasing hormone agonist (GnRHa)-induced pituitary suppression. The molecular mechanisms involved were also studied using tissues from endometrial biopsies. Patients who underwent FET were assigned to 5 groups as follows: Group A underwent a mNC ( $n=276)$; group $B(n=338)$ received estradiol (E2) and progesterone (P4); group $\mathrm{C}$ received 1 cycle of GnRHa, E2 and P4 $(n=323)$; group D received 2 cycles of GnRHa, E2 and P4 $(n=329)$; and group E received 3 cycles of GnRHa, E2 and P4 $(n=323)$. Tissues from endometrial biopsies of 91 patients performed on the day of ET were tested for endometrial receptivity marker mRNA expression and microRNA (miR)-223-3p mRNA. Furthermore, endometrial stromal cells (ESCs) were used for an in-depth study of the molecular mechanisms involved. Among the 5 groups of patients, implantation rates, clinical pregnancy rates and live birth rates were not
\end{abstract}

Correspondence to: Professor Xuehong Zhang, The Reproductive Medicine Special Hospital of The First Hospital of Lanzhou University, 1 Donggang West Road, Chengguan, Lanzhou, Gansu 730000, P.R. China

E-mail: zhangxueh@lzu.edu.cn

Abbreviations: CPR, clinical pregnancy rate; E2, estradiol; ESC, endometrial stromal cell; ET, embryo transfer; FET, frozen-thawed embryo transfer; FHB; fetal heartbeat; GnRHa, gonadotropin-releasing hormone agonist; HT, hormonal therapy cycle; IVF, in vitro fertilization; IR, implantation rate; LBR, live birth rate; $\mathrm{mNC}$, modified natural cycle; $\mathrm{P} 4$, progesterone

Key words: FET, endometrial receptivity, GnRHa, leukemia inhibitory factor, decidualization significantly different. However, endometrial receptivity was enhanced in group $\mathrm{E}$ when compared with groups A-D, which was associated with endometrial leukemia inhibitory factor (LIF), osteopontin, vascular endothelial growth factor, integrin $\beta 3$ and homeobox gene 10 and 11 mRNA upregulation, and miR-223-3p miRNA downregulation. Transfection of ESCs with an miR-223-3p mimic significantly reduced levels of LIF mRNA and protein. In addition, pre-treating ESCs with GnRHa upregulated mRNA and protein expression of the decidualization markers prolactin and insulin-like growth factor binding protein-1 in a time-dependent manner. In conclusion, these results indicated that HT with GnRHa may be a potential endometrial preparation protocol for FET.

\section{Introduction}

Since the first successful frozen-thawed embryo transfer (FET) was reported in 1983 (1), embryo cryopreservation has become an important issue. FET is used as a complementary treatment with stimulated in vitro fertilization (IVF) cycles in patients with infertility and has attracted increasing attention worldwide (2). FET cycles offer certain advantages to patients, including higher cumulative pregnancy rates and lower cost compared with fresh embryo transfer cycles (3). Furthermore, IVF-associated complications, such as ovarian hyperstimulation syndrome and multifetal gestation, can be effectively prevented using FET (4).

Optimal endometrial receptivity and synchronization between embryonic and endometrial development serve important roles in successful pregnancies resulting from FET cycles (5). Several approaches to prepare the endometrium have been proposed, including natural cycles (NCs) and hormone therapy cycles (HTs), which can be performed with or without pituitary gland suppression induced by gonadotropin-releasing hormone agonist (GnRHa). The main benefits of $\mathrm{NC}$ are reduced cost and medication requirements. Performing HT without GnRHa avoids the cost of GnRHa, while HT with GnRHa enables good control of the timing of cycles as complete pituitary suppression and effective cycle control are achieved by the use of GnRHa (6). However, HT with GnRHa is the most labor-intensive, time-consuming and expensive method (7). 
Although certain previous studies have compared different protocols for FET, the results have been conflicting. Certain investigations reported better pregnancy outcomes using HT-FET with GnRHa, while others indicated the superiority of HT-FET without GnRHa (8-10). Additionally, certain retrospective studies have demonstrated comparable or higher clinical pregnancy rates (CPR), clinical pregnancy with fetal heartbeat $(\mathrm{FHB})$ rates and live birth rates (LBR) in patients who underwent NC compared with those who underwent HT with GnRHa (11-14). It should be noted that only 1 or 2 cycles of GnRHa were used in all these previous studies. To the best of our knowledge, the mechanism of action of GnRHa on the endometrium in FET cycles has not previously been studied; however, the effects of GnRHa on endometrial nitric oxide synthase and $\alpha v \beta 3$ integrin expression in fresh ET cycles have been reported $(15,16)$. Numerous factors are involved during embryo implantation. Proteins expressed during the window of implantation (WOI) act as biomarkers of endometrial receptivity (17). Leukemia inhibitory factor (LIF), vascular endothelial growth factor (VEGF), homeobox A11 (HOXA10), homeoboxA11 (HOXA11), integrin $\beta 3$, and osteopontin (OPN) are widely regarded as markers of endometrial receptivity (18-24).

MicroRNAs (miRs/miRNAs) are small non-coding RNAs that function as transcriptional regulators of gene expression and have been widely reported to be involved in embryo implantation (25). In mice, low levels of miR-223-3p during the WOI are essential for initiating implantation as they downregulate LIF expression (25). Decreased expression of miR-223-3p also promoted the formation of pinopodes, membrane protrusions on the apical surface of non-ciliated endometrial epithelial cells during the secretory phase, which may serve as the preferred attachment sites for embryos (25-27). However, the relationship between miR-223-3p and human endometrial receptivity has not yet been determined.

Decidualization of the endometrium is essential for successful implantation of all species and characterized by transformation of endometrial stromal cells (ESCs) into secretory decidual cells (28). Major secretory products of decidual stromal cells include prolactin (PRL) and insulin-like growth factor binding protein-1 (IGFBP-1), two proteins that have been used as markers of decidualization (29). However, to the best of our knowledge, few reports have investigated the effect of GnRHa on human endometrial decidualization.

The current study aimed to evaluate different protocols for endometrial preparation for FET in regularly menstruating patients with infertility, including modified NC (mNC), HT without GnRHa and HT with 1-3 cycles of GnRHa. CPR, miscarriage rates and LBR resulting from these FET cycles were compared between groups. Furthermore, the molecular mechanisms involved were investigated.

\section{Materials and methods}

Patients. The current retrospective study was conducted at the Reproductive Medicine Special Hospital of the First Hospital of Lanzhou University (Lanzhou, China). A total of 1,589 women (age range, $21-40$ years; mean age, $33.43 \pm 4.45$ years) who underwent FET cycles between January 2015 and December 2018 were included.
Patients who underwent FET, were aged 21-40 years, had regular menstrual cycles (length, 24-35 days) and had at least 2 cryopreserved embryos were included. Patients who underwent FET following pre-implantation genetic testing or had endometriosis, uterine malformations, hydrosalpinx and/or a history of intrauterine adhesions were excluded.

Endometrial preparation protocols. Group A $(n=276)$ underwent mNCs. Ultrasonic monitoring of the dominant follicle was performed on days 3 and 10-12 of the menstrual cycle. When the mean diameter of the dominant follicle was $\geq 17 \mathrm{~mm}$ and the endometrial thickness was $\geq 7 \mathrm{~mm}$, human chorionic gonadotropin (hCG; 5,000 IU Pregnylor $250 \mathrm{mg}$ Ovitrelle; Merck KGaA) was injected to trigger ovulation. ET was conducted 3 days (cleavage stage embryos) or 5 days (blastocyst stage embryos) later. Embryo quality was graded as 'good', 'reasonable', 'moderate' or 'poor' according to renewed development of the embryo, degree of fragmentation and number of cells using the manual scoring system of the Leuven University Fertility Centre (30). No luteal support was provided.

Group B underwent HT without GnRHa $(n=338)$. Oral E2 (2 mg Progynova 3 times/day; Bayer AG) was administered on day 3 of the menstrual cycle. Ultrasound was performed after 7-8 days to confirm that no dominant follicle had emerged and to measure endometrial thickness. If no dominant follicle was present and the endometrial thickness was $\geq 7 \mathrm{~mm}$, P4vaginal suppositories (600 mg Utrogestan daily; Besins Healthcare) were administered and FET was scheduled. ET was performed after 3 days (cleavage-stage embryos) or 5 days (blastocyst-stage embryos) post-P4 administration. From the day of ET, P4vaginal suppositories (400 mg Utrogestan2 times/day; Besins Healthcare) were administered for luteal support. If pregnancy was confirmed, luteal support was continued for the first 12 weeks of pregnancy.

Groups C-E underwent HT with GnRHa. Ovulation was confirmed by transvaginal ultrasound and GnRHa $(1.875 \mathrm{mg}$ leuprorelin; Livzon Pharmaceutical Group Inc.) was injected at the luteal phase (1 week post-ovulation). Injections continued for 1 cycle (group $C ; n=323$ ), 2 cycles (group D; $n=329$ ) or 3 cycles (group $E ; n=323$ ), with each cycle of injections lasting 3 weeks. Oral E2 (2 mg Progynova 3 times/day; Bayer AG) was administered from day 3 of the menstrual cycle and the HT protocol was then performed as in group B.

ET and outcomes. ET was performed using the same method for all 5 groupsaccording tothe Hillmethod (13). For each patient, 2 or 3 viable and high-grade frozen-thawed embryos were transferred. The embryo laboratory used the original criteria for embryo quality and the current study adopted these criteria to make embryo quality more accurate (30).

At 14 days post-ET, serum $\beta$-hCG, E2 and P4 levels (data not shown) were measured to assess pregnancy, and ultrasonography was performed to confirm the number of gestational sacs and fetal viability. The primary outcome was clinical pregnancy. Implantation was defined as the presence of a gestational sac and clinical pregnancy as the detection of an embryonic heartbeat at $\sim 6$ weeks of gestation. Clinical pregnancy with FHB was defined as the presence of a fetus in the uterus with a positive FHB at 12 weeks of gestation. 
Miscarriage was confirmed by transvaginal ultrasonography and a decrease in serum $\beta$-hCG level. LB was defined as the delivery of a live baby at $\geq 24$ weeks of gestation.

Samples. Ethics approval for tissue collection was obtained from the Institutional Ethics Committee of the First Hospital of Lanzhou University (Lanzhou, China). Endometrial biopsies were obtained using an endometrial sampler (Endocell) from patients whose ETs were cancelled due to lack of transplantable embryos or for personal reasons. The samples were obtained on the day the ET had been scheduled. The baseline characteristics of patients whose endometrial biopsy samples were obtained are shown in Table SI.

Endometrial biopsy specimens taken from the uterine fundus were washed with sterile PBS and samples were either immediately preserved in liquid nitrogen until subsequent analysis or fixed in formalin at room temperature for immunohistochemical analysis. Immunohistochemical analysis was performed as previously described (31).

Endometrial stromal cell culture. An ESC line was purchased from the American Type Culture Collection (cat. no. CRL-4003). ESCs were cultured in DMEM/Nutrient Mixture F-12 containing 1\% (v/v) penicillin/streptomycin and $10 \%$ (v/v) FBS (all Gibco; Thermo Fisher Scientific, Inc.). The cells were cultured in $5 \% \mathrm{CO}_{2}$ at $37^{\circ} \mathrm{C}$ in a humidified atmosphere. Mycoplasma testing was performed with a One-step Quickcolor Mycoplasma Detection kit (Clark Bioscience) for the cell line used and there was no mycoplasma infection present. Cells culture supernatants were tested when cells reached $80-90 \%$ confluence. A total of $46 \mu \mathrm{l}$ solution 1 and $2 \mu 1$ solution 2 were mixed and divided into two pipes. A total of $1 \mu \mathrm{l}$ mycoplasma DNA was added into the positive tube and $1 \mu \mathrm{l}$ cell culture supernatant was added into the test tube. Subsequently, $25 \mu \mathrm{l}$ mineral oil was added to each tube and the tubes were placed in a water-bath at $61^{\circ} \mathrm{C}$ for $1 \mathrm{~h}$. A solution color of purple-red indicated no mycoplasma was present in the sample. Additionally, the cell line used was authenticated by immunohistochemistry (32).

Endometrial stromal cell decidualization. ESCs were pre-treated with $0.7 \mu \mathrm{M}$ GnRHa (Sigma-Aldrich; Merck $\mathrm{KGaA}$ ) for $0,24,48$ or 72 h. Opti-MEM ${ }^{\circledR}$ I Reduced Serum medium containing 2\% FBS (Gibco; Thermo Fisher Scientific, Inc.), $95 \mathrm{nM}$ estrogen (Sigma-Aldrich; Merck KGaA), $9 \mu \mathrm{M}$ progesterone (Sigma-Aldrich; Merck KGaA) and $50 \mu \mathrm{M}$ cyclic adenosine monophosphate (Sigma-Aldrich; Merck KGaA) were added to initiate decidualization. The medium was replaced every 2 days for 2 weeks. Cells were then harvested and mRNA and protein expression of the decidualization markers PRL and IGFBP-1 were tested using quantitative PCR (qPCR) and western blotting to confirm in vitro decidualization.

RNA extraction and reverse transcription $q P C R(R T-q P C R)$ analysis. RNA was isolated from endometrial biopsy specimens or ESCs using a TRNzol A+ reagent kit (Tiangen Biotech Co., Ltd.) and reverse transcribed using a FastKingg DNA Dispelling RT SuperMix kit (Tiangen Biotech Co., Ltd), according to the manufacturer's protocol. RT-qPCR was conducted using a SYBR Premix Ex Taq kit (Tiangen
Biotech Co., Ltd) and a real-time ViiA 7 Real-Time PCR system (Applied Biosystems; Thermo Fisher Scientific, Inc.). Relative gene expression levels were analyzed according to the $2^{-\Delta \Delta \mathrm{Cq}}$ method (33). Primers for leukemia inhibitory factor (LIF), osteopontin (OPN), vascular endothelial growth factor (VEGF), integrin $\beta 3$, homeobox genes (HOXA10 and HOXA11), and $\beta$-actin were obtained from Thermo Fisher Scientific, Inc. The thermocycling conditions were $95^{\circ} \mathrm{C}$ for $15 \mathrm{~min}$, followed by 40 cycles at $95^{\circ} \mathrm{C}$ for $10 \mathrm{sec}$ and $65^{\circ} \mathrm{C}$ for $20 \mathrm{sec}$. The primers sequences were as follows: LIF forward, 5'-TGGTTCTGCACTGGAAACATG-3' and reverse, 5'-GTA ATAGAGAATAAAGAGGGCATTGG-3'; OPN forward, 5'-ACCCTTCCAAGTAAGTCCAACG-3' and reverse, 5'-GGT GAGAATCATCAGTGTCATCTAC-3'; VEGF forward, 5'-ATTCAACGGACTCATCAGCCA-3' and reverse, 5'-GCA ACCTCTCCAAACCGTTG-3'; HOXA10 forward, 5'-AGG TGGACGCTGCGGCTAATCTCTA-3' and reverse, 5'-GCC CCTTCCGAGAGCAGCAAAG-3'; HOXA11 forward, 5'-TCC AGCCTCCCTTCTTTTTTG-3' and reverse, 5'-GTAGCA GTGGGCCAGATTGC-3'; integrin $\beta 3$ forward, 5'-GGCGTT GTTGTTGGAGAGTC-3' and reverse, 5'-GCCTCACTGACT GGGAACTC-3'; PRL forward, 5'-CACTACATCCATAAC TCTC-3' and reverse, 5'-ATGCTGACTATCAAGCTCAG-3'; IGFBP-1 forward, 5'-TATGATGGGGCTCGAAGGTCT C-3' and reverse, 5'-GTAGACGCACCAGCAGAGTC-3'; and $\beta$-actin forward, 5'-CGTACCACTGGCATCGTGAT-3' and reverse, 5'-GTGTTGGCGTACAGGTCTTTG-3'.

Furthermore, poly(A) RT-qPCR was performed using a miRNA First-Strand cDNA synthesis kit (Tiangen Biotech Co., Ltd.) for miRNA detection of ESCs following RNA extraction. The reverse transcription conditions were as follows: $42^{\circ} \mathrm{C}$ for $1 \mathrm{~h}$, followed by $95^{\circ} \mathrm{C}$ for $3 \mathrm{~min}$. A miRcute Plus miRNA qPCR assay kit (Tiangen Biotech Co., Ltd.) was used to examine miR-223-3p expression. The thermocycling conditions were $95^{\circ} \mathrm{C}$ for $15 \mathrm{~min}$, followed by 40 cycles of $94^{\circ} \mathrm{C}$ for $20 \mathrm{sec}$ and $60^{\circ} \mathrm{C}$ for 34 sec. miR-223-3p and U6 snRNA primers were purchased from Tiangen Biotech Co., Ltd. $\beta$-actin and U6 were used as internal references for mRNA and miRNA, respectively. The following primer pairs were used: miR-223-3p forward, 5'-GGTACCCAAAGTCAA CTACTTTCTTCTCCCTT-3' and reverse, 5'-CTCGAGCCA GATGGAATTGGGCTTT-3' and U6 forward, 5'-CTTCGG CAGCACATATAC-3' and reverse, 5'-GAACGCTTCACG AATTTGC-3'. Relative gene expression levels were analyzed according to the $2^{-\Delta \Delta \mathrm{Cq}}$ method (33).

Cell transfection. LIF was predicted to be a direct target of miR-223-3p by TargetScan (v7.2; targetscan.org). Mimics and corresponding negative controls of miR-223-3p were synthesized by Shanghai GenePharma Co., Ltd. A total of $2 \times 10^{5} \mathrm{ESCs} /$ well were plated in six-well plates and miR-223-3p mimics or negative controls were transfected into cells using a transfection kit (RFects RNA transfection reagent; Bioline; Meridian Bioscience) when they reached $50 \%$ confluence. The final concentration of miR-223-3p mimics or negative controls was $50 \mathrm{nM}$. RNA and protein samples were collected after $48 \mathrm{~h}$. he negative control is a generic sequence and has no homology with any mammal. The primer sequences were as follows: miR-223-3p mimics forward, 5'-UGUCAGUUUGUC AAAUACCCCA-3' and reverse, 5'-GGGUAUUUGACAAAC 
UGACAUU-3' and negative control forward, 5'-UUCUCC GAACGUGUCACGUTT-3' and reverse, 5'-ACGUGACAC GUUCGGAGAATT-3'.

Western blotting. Total protein from the endometrium and ESCs was extracted using a RIPA lysis buffer containing protease and phosphatase inhibitor cocktails (allBIOSS). Protein concentration was determined using a spectrophotometer (NanoDrop ${ }^{\mathrm{TM}}$ 1000; Thermo Fisher Scientific, Inc.). SDS-PAGE (12\% gel) was used to separate $50 \mu \mathrm{g}$ total protein/lane and then transferred to PVDF membranes. The membranes were blocked with $5 \%$ non-fat milk dissolved in Tris-buffered saline (2 mMTris- $\mathrm{HCl}$, $140 \mathrm{mMNaCl}$; pH 7.6) with $1 \%$ Tween-20 (TBST) for $1 \mathrm{~h}$ at room temperature and then probed with corresponding polyclonal rabbit antibodies against LIF (cat. no. bs-1058R), PRL (cat. no. bs-0508R), IGFBP-1 (cat. no. bs-1822R) and $\beta$-actin (cat. no. bs-10966R) at $4^{\circ} \mathrm{C}$ overnight. All of the primary antibodies were purchased from BIOSS and diluted at 1:500. Following 5 washes with TBST, membranes were incubated with goat anti-rabbit immunoglobulin G H\&L horseradish peroxidase-conjugated secondary antibodies (cat. no. bs-40295G-HRP; 1:5,000; BIOSS) for $1 \mathrm{~h}$ at room temperature. Target protein signals were visualized using an enhanced chemiluminescence detection system (Model42i-TL TRACE Level Nox analyzer; Thermo Fisher Scientific, Inc.).

Statistical analysis. Statistical analysis was performed using SPSS software (version 24.0; IBM Corp.). Data are presented as the mean $\pm \mathrm{SD}$ or number $(\%)$ for triplicate measurements. For quantitative variables (means of continuous parametric data), comparisons between groups were analyzed using one-way ANOVA followed by Tukey's honest significant difference post-hoc test. $\mathrm{P}<0.05$ was considered to indicate a statistically significant difference. For qualitative variables (rates), the $\chi^{2}$ test followed by Bonferroni's correction was performed to test differences between groups. Comparisons of gene expression between two groups of cells were made using Student's t-test. Homogeneity of variance and normality of data were estimated using Levene and Kolmogorov-Smirnov tests, respectively. $\mathrm{P}<0.005$ was considered to indicate a statistically significant difference in multiple group comparisons.

\section{Results}

Demographic characteristics and clinical outcomes. Patients' baseline characteristics are displayed in Table I. No statistically significant differences were found between groups with regard to basic characteristics, such as age, body mass index, duration of infertility, fertility status or previous fresh ET cycles. The etiology of infertility, mean number of embryos transferred, embryo quality scores, developmental stage at cryopreservation, duration of cryopreservation, embryo survival rates and endometrial thicknesses were also similar among groups. This suggests that the groups had comparable pregnancy potential.

Reproductive outcomes are shown in Table II. IR (UI+EU) and IR with FHB, LBR per embryo transferred and CPR (UI+EU) per embryo transfer cycle were significantly different between groups $(\mathrm{P}<0.005)$. No significant differences were reported between groups for CPR with FHB and LBR per embryo transfer cycle $(\mathrm{P}>0.005)$. Additionally, miscarriage rate per embryo transferred and per embryo transfer cycle were not significantly different between groups $(\mathrm{P}>0.005)$. It should be noted that there no stillborns were found in the $\mathrm{HT}+3 \mathrm{GnRHa}$ group, while one stillborn was found in both $\mathrm{mNC}$ and $\mathrm{HT}+2 \mathrm{GnRHa}$ groups and there were 2 stillborns in both HT and HT+1GnRHa groups.

Comparison of endometrial expression of LIF, integrin $\beta 3$, OPN, VEGF, HOXA10 and HOXA11 mRNA between groups. Baseline characteristics of subjects whose endometrial specimens were collected are shown in Table SI. RT-qPCR was performed to determine expression levels of endometrial receptivity markers present in endometrial specimens on the day of embryo implantation from patients who did not undergo ET. Endometrial LIF and integrin $\beta 3$ mRNA expression levels were significantly upregulated in group $\mathrm{E}$ compared with $\mathrm{mNC}$ and HT groups (Fig. 1). However, OPN, VEGF, HOXA10 and HOXA11 mRNA levels in group E were not significantly increased compared with those in other groups. Furthermore, western blotting demonstrated that endometrial LIF protein in group E was higher compared with that in group A (Fig. S1).

miR-223-3p interacts with the 3'-untranslated region (3'-UTR) of LIF and regulates LIF expression in human endometrial cells. miR-223-3p miRNA levels were assessed in the 5 groups. miR-223-3p levels were significantly decreased in group E compared with groups A and B (Fig. 2A and B). These results contrasted with the aforementioned pattern of LIF and integrin $\beta 3$ mRNA levels.

Human LIF was predicted to be regulated by miR-223-3p according to TargetScan (targetscan.org). miR-223-3p can directly interact with human LIF through its 3 '-UTR (Table SII). In order to determine the effect of miR-223-3p on LIF, miR-223-3p mimics and corresponding negative controls were transfected into ESCs. miR-223-3p was upregulated $\sim 3.8$-fold in the miR-223-3p mimic group $(1.0 \pm 0.5$ vs. $3.83 \pm 0.5$; $\mathrm{P}<0.05$; Fig. $2 \mathrm{~B})$ and LIF mRNA levels decreased by $\sim 60 \%(1.0 \pm 0.34$ vs. $0.38 \pm 0.22, \mathrm{P}<0.05$; Fig. $2 \mathrm{C})$ compared with that in the control group. LIF protein levels also decreased following treatment with miR-223-3p (Fig. 2D).

GnRHa induces ESC decidualization. The effect of GnRHa on the decidualization of ESCs was analyzed. Pre-treatment of ESCs with GnRHa increased PRL and IGFBP-1 mRNA levels (Fig. 3A) and protein levels (Fig. 3B) in a time-dependent manner.

\section{Discussion}

Numerous studies have compared endometrial preparation protocols for FET (NC, HT with or without GnRHa). However, few studies have performed a full analysis and overall comparison of the three protocols. Additionally, to the best of our knowledge, GnRHa was administered for only 1 or 2 cycles in all previous studies and none investigated the molecular mechanisms involved (1-11). Therefore, the current study aimed to analyze the clinical outcomes of FET with mNC, HT without GnRHa or HT with GnRHa in patients with infertility and regular menstrual cycles. 
Table I. Baseline characteristics of all ET cycles.

Type of endometrial preparation

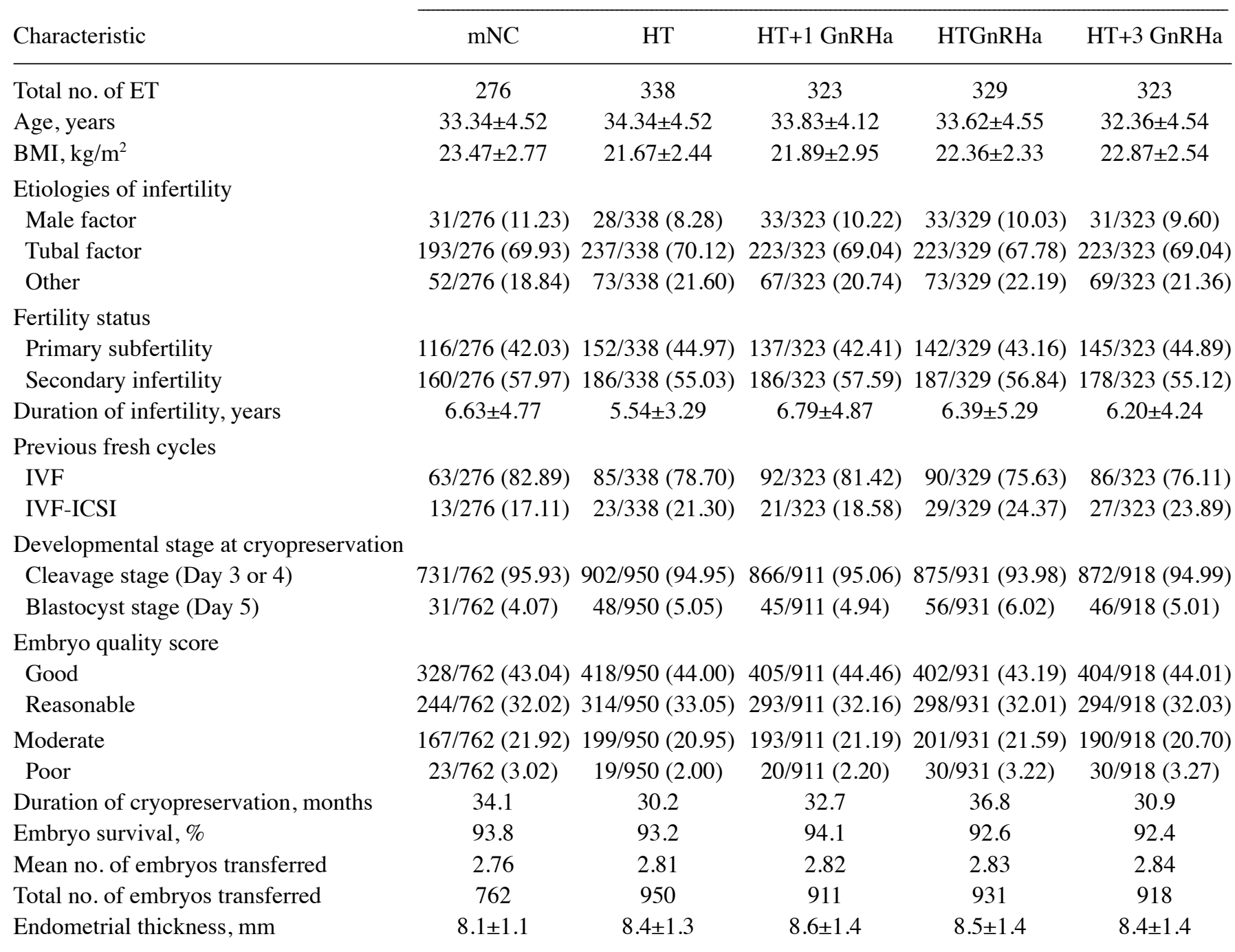

Data are presented as the mean $\pm \mathrm{SD}, \mathrm{n} /$ total $(\mathrm{n} \%)$ or mean. 1, 2 and 3 refer to the number of cycles of GnRHa, E2 and P4 treatment; mNC, modified natural cycle; HT, hormone therapy cycle; GnRHa, gonadotropin-releasing hormone agonist; ET, embryo transfer; BMI, body mass index; IVF, in vitro fertilization; IVF-ICSI, in vitro fertilization and intracytoplasmic sperm injection.

The results demonstrated that pregnancy outcomes were better in group E; however, there were no significant differences in CPR or LBR when compared with the other groups. However, molecular changes were observed, including increased expression of endometrial receptivity and decidualization markers in patients who underwent HT with GnRHa.

To the best of our knowledge, the current study is the first to compare clinical outcomes and expression of endometrial receptivity markers in patients who underwent FET with $\mathrm{mNC}$ and HT with and without GnRHa. Furthermore, the present study was the first to investigate HT-FET with different numbers of GnRHa cycles. Certain previous studies have assessed HT with and without GnRHa pituitary suppression. Dal Prato et al (7) and Simon et al (11) demonstrated that similar pregnancy outcomes resulted from FET cycles using HT with or without GnRHa. Another review indicated that all endometrial preparation protocols are equally effective with respect to CPR, CPR with FHB or LBR (14). However, a single prospective clinical trial demonstrated that HT without GnRHa led to a lower pregnancy rate compared with mNC cycles (13). Furthermore, another review indicated that HT-FET with GnRHa resulted in a higher LBR compared with HT-FET without GnRHa (3). However, a retrospective study of 187 women reported that, although pregnancy outcomes were better in the GnRHa group, IR and LBR were not significantly different to those of the HT-FET group (16). These varying results indicate that concrete conclusions cannot be drawn from previous studies. Furthermore, only 1 or 2 cycles of GnRHa were administered in these previous studies. Therefore, the current study compared HT-FET with different numbers of cycles of GnRHa as well as HT-FET without GnRHa and mNC-FET.

Although baseline characteristics, embryo survival and mean number of embryos transferred were similar between 
Table II. Clinical outcomes of frozen-thawed ETs.

Type of endometrial preparation

\begin{tabular}{lcccccr}
\cline { 2 - 5 } Characteristic & $\mathrm{mNC}$ & $\mathrm{HT}$ & $\mathrm{HT}+1 \mathrm{GnRHa}$ & $\mathrm{HT}+2 \mathrm{GnRHa}$ & $\mathrm{HT}+3 \mathrm{GnRHa}$ & P-value \\
\hline $\begin{array}{l}\text { Reproductive outcome per embryo } \\
\text { transferred }\end{array}$ & & & & & & \\
Total no. of embryos transferred & 762 & 950 & 911 & 931 & 918 & \\
IR (IU+EU) & $118(15.49)$ & $139(14.63)$ & $176(19.32)$ & $201(21.59)$ & $208(22.66)$ & $<0.001$ \\
IR with FHB & $99(12.99)$ & $119(12.53)$ & $156(17.12)$ & $177(19.01)$ & $186(20.26)$ & $<0.001$ \\
Miscarriage rate & $15(15.15)$ & $19(15.97)$ & $27(17.31)$ & $29(16.38)$ & $33(17.46)$ & 0.985 \\
LBR & $83(10.89)$ & $98(10.32)$ & $127(13.94)$ & $147(15.79)$ & $153(16.67)$ & $<0.001$ \\
Reproductive outcome per ET cycle & & & & & & \\
Total no. ofembryos transferred & 276 & 338 & 323 & 329 & 323 & \\
CPR (IU+EU) & $86(31.16)$ & $107(31.66)$ & $119(36.84)$ & $138(41.95)$ & $144(44.58)$ & 0.001 \\
CPR with FHB & $75(27.17)$ & $94(27.81)$ & $107(33.13)$ & $122(37.08)$ & $131(40.56)$ & 0.027 \\
Miscarriage rate & $13(17.33)$ & $18(19.15)$ & $17(15.89)$ & $21(17.21)$ & $23(17.56)$ & 0.959 \\
LBR & $62(22.46)$ & $76(22.49)$ & $90(27.86)$ & $101(30.70)$ & $108(33.44)$ & 0.021
\end{tabular}

Data are presented as number (\%). 1, 2 and 3 refer to the number of cycles of GnRHa, E2 and P4 treatment; mNC, modified natural cycle; HT, hormone therapy cycle; GnRHa, gonadotropin-releasing hormone agonist; IR, implantation rate; IU, intrauterine; EU, extrauterine; FHB, fetal heartbeat; ET, embryo transfer; CPR, clinical pregnancy rate; LBR, live birth rate.
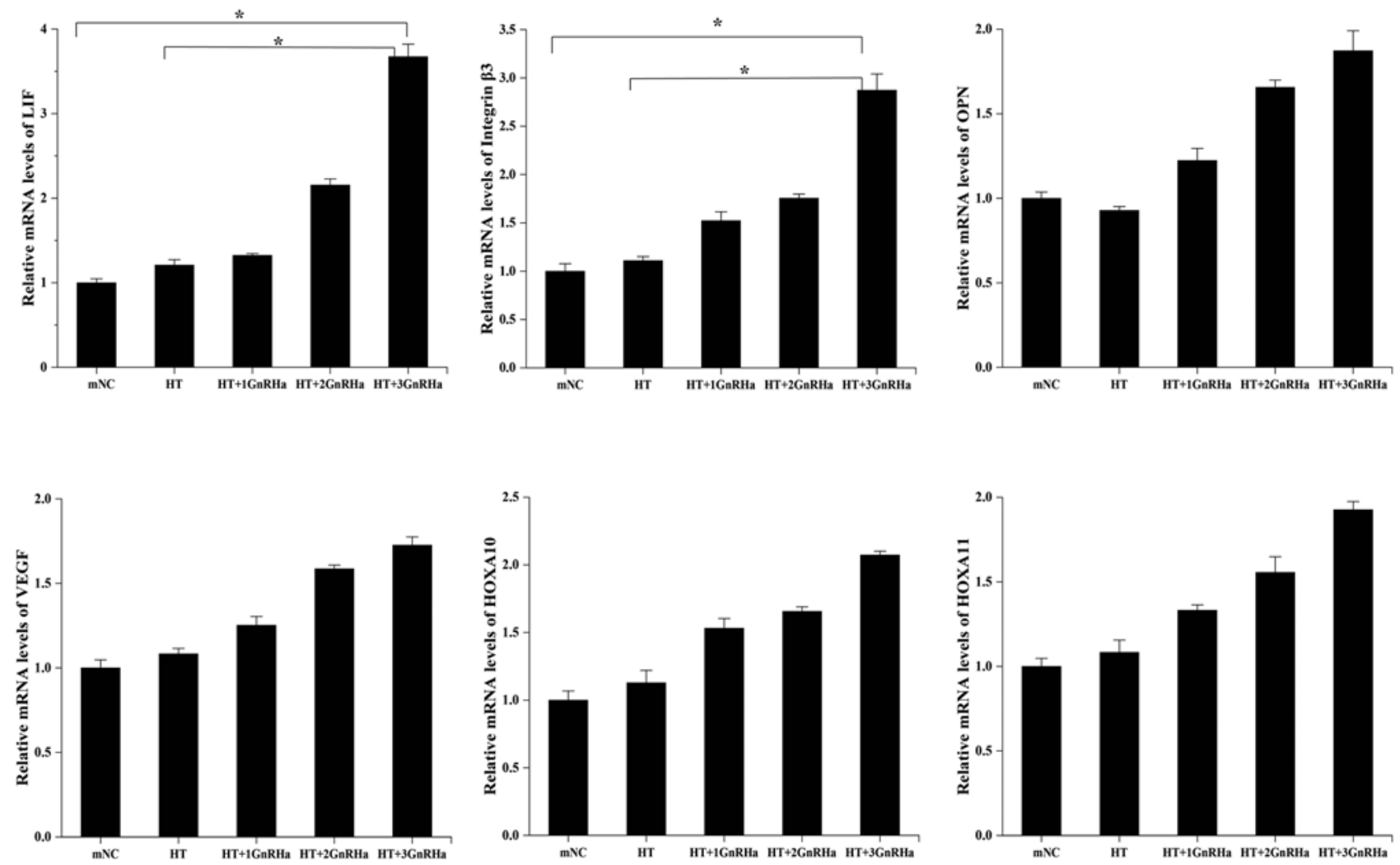

Figure 1. Quantitative analysis of mean endometrial LIF, integrin $\beta 3$, OPN, VEGF, HOXA10and HOXA11mRNA levels of patients who did not undergo embryo transfers on the day of embryo transfer. "P<0.05. 1, 2 and 3 refer to the number of cycles of GnRHa, E2 and P4 treatment; LIF, leukemia inhibitory factor; OPN, osteopontin; VEGF, vascular endothelial growth factor; HOXA10 and 11, homeobox genes 10 and 11; mNC, modified natural cycle; HT, hormone therapy cycle; GnRHa, gonadotropin-releasing hormone agonist.

groups, the HT with GnRHa groups had higher IR, CPR and LBR compared with the mNC group. Kang et al (17) compared
88 cycles of HT-FET without GnRHa and 181 cycles of HT-FET with GnRHa. Similar to the results of the current study, this 
A

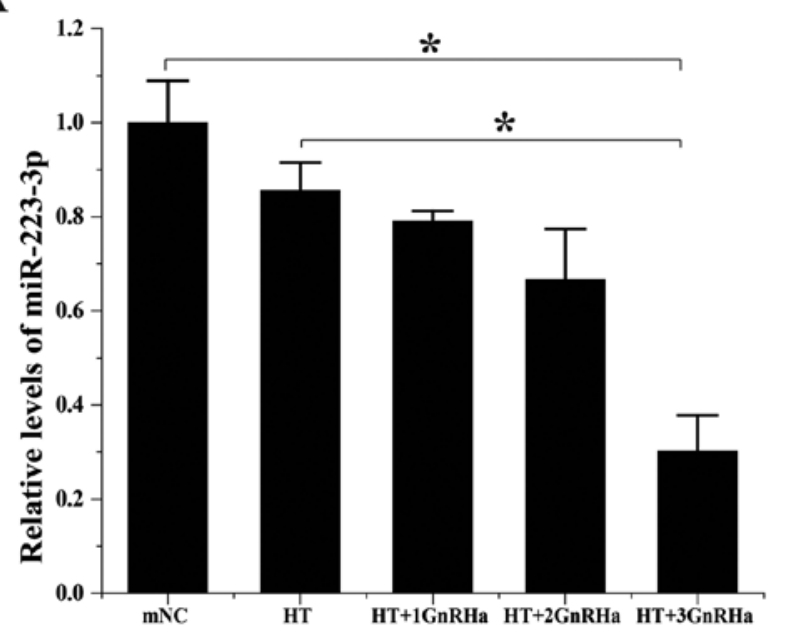

$\mathbf{B}$

C

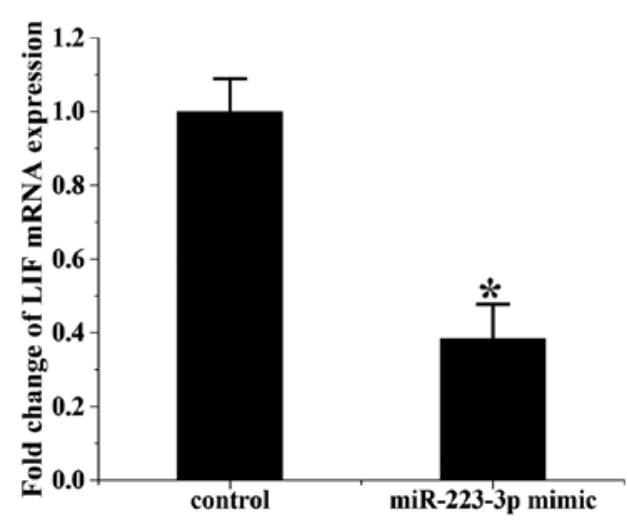

D
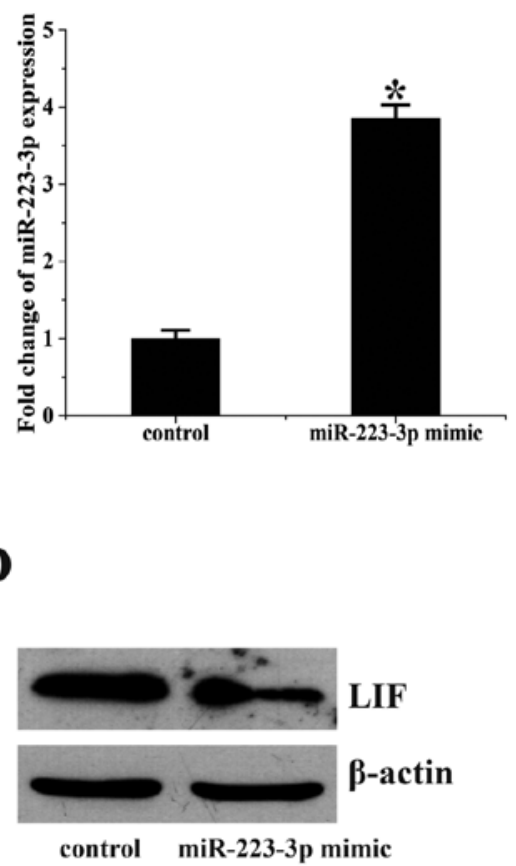

Figure 2. miR-223-3p overexpression suppresses LIF expression. (A) miR-223-3p expression in the endometrium on the day of embryo transfer. "P $<0.05$. (B) miR-223-3p expression in ESCs treated with miR-223-3p mimics or hsa-miR negative controls. Transfection with miR-223-3p mimics significantly increased the expression of miR-223-3p. (C) LIF mRNA expression in ESCs treated with hsa-miR-223-3p mimics or negative control. (D) LIF protein expression levels following treatment with miR-223-3p mimics or negative controls were analyzed via western blotting. ${ }^{*} \mathrm{P}<0.05$ vs. control. 1,2 and 3 refer to the number of cycles of GnRHa, E2 and P4 treatment; miR, microRNA; LIF, leukemia inhibitory factor; ESC, endometrial stromal cell; ESC, endometrial stromal cell; mNC, modified natural cycle; HT, hormone therapy cycle; GnRHa, gonadotropin-releasing hormone agonist.

A

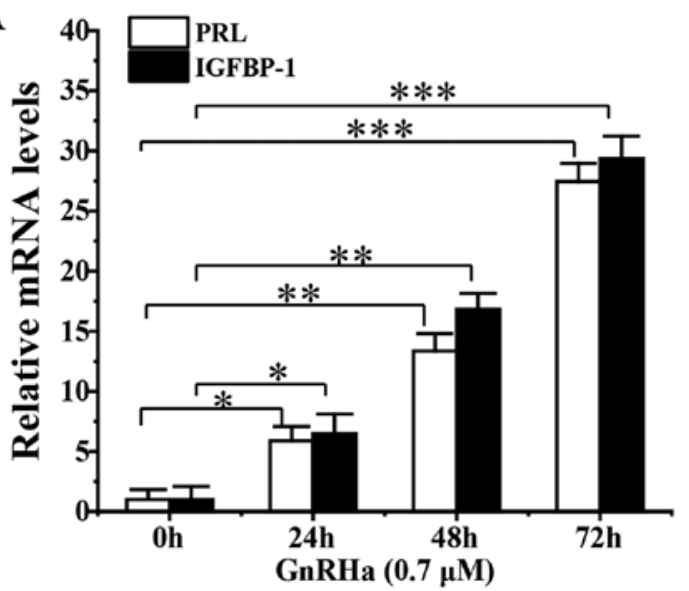

B

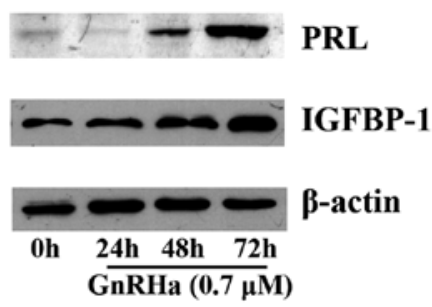

Figure 3. Effect of GnRHa on decidualization of ESCs.(A) mRNAand (B) protein levels following decidualization of ESCs pre-treated with $0.7 \mu \mathrm{M}$ GnRHa for $0,24,48$ or $72 \mathrm{~h} .{ }^{*} \mathrm{P}<0.05,{ }^{* *} \mathrm{P}<0.01,{ }^{* * * *} \mathrm{P}<0.005$. GnRHa, gonadotropin-releasing hormone agonists; ESC, endometrial stromal cell; PRL, prolactin; IGFBP-1, insulin-like growth factor binding protein-1.

study reported a higher CPR (52.2 vs. 42.9\%) and LBR (41.6 vs. $32.7 \%)$ and a lower miscarriage rate (12.7 vs. $20.0 \%)$ in the HT with GnRHa group compared with the HT without GnRHa group (13). Similarly, a prospective randomized trial 
compared 117 women who underwent HT-FET with GnRHa and 117 women who underwent HT-FET without GnRHa and reported a higher CPR (24.0 vs. $11.3 \%)$ and LBR (20.0 vs. $8.5 \%$ ) in the GnRHa group (8).

To the best of our knowledge, the mechanism of action of GnRHa on the endometrium in FET cycles has not previously been studied; however, the effects of GnRHa on endometrial nitric oxide synthase and $\alpha v \beta 3$ integrin expression in fresh ET cycles have been reported $(23,24)$.

In the current study, levels of miR-223-3p decreased by more than half and the expression of LIF, integrin $\beta 3$, OPN, VEGF, HOXA10, and HOXA11 mRNA increased in group E compared with the other groups, indicating that miR-223-3p may be an inhibitor for embryo implantation. In order to evaluate the specificity of miR-223-3p/LIF regulation, the human ESC line was transfected with miR-223-3p mimics and negative controls to determine whether miR-223-3p regulated LIF in a reproductive tract cell line. LIF expression was significantly downregulated by miR-223-3p overexpression, indicating that miR-223-3p regulated LIF in the human endometrium. miRNAs regulate numerous biological processes by pairing with the 3'-UTRs of target mRNAs and inhibiting their translation (27). To confirm whether miR-223-3p was capable of directly regulating LIF in the human endometrium, the 3'-UTR of LIF was investigated and a site was discovered that could be recognized by miR-223-3p. Furthermore, the results demonstrated that miR-223-3p directly targeted the 3'-UTR of LIF, suppressing LIF expression. These results indicated that miR-223-3p might inhibit LIF expression during embryo implantation in humans. Moreover, miR-223-3p may be a biomarker for endometrial receptivity.

Decidualization is an important event in human pregnancy (34-37). Abnormal decidualization of endometrial stromal cells has been correlated with unexplained infertility, miscarriage andendometrial pathologies such as endometriosis (34-37). PRL and IGFBP-1 act as markers of decidualization (38). A 2009 study demonstrated that GnRH analogues had no significant effect on the decidualization of ESCs (39). However, this previous study administered GnRH analogues during decidualization, rather than beforehand. By contrast, the current study administered GnRH analogues prior to decidualization and ESCs were pre-treated with GnRHa for $0,24,48$ or $72 \mathrm{~h}$. The results demonstrated that GnRHa induced decidualization in a time-dependent manner. Therefore, GnRHa may affect decidualization of the endometrium.

While the current study provides information that may be used for clinical treatment, it had certain limitations. Firstly, the retrospective nature of the current study indicates that there may have been certain confounding factors, such as additional drugs administered for implantation, such as atosiban, aspirin and lipid emulsion, which may have affected the outcomes of FET. Secondly, patients with polycystic ovary syndrome and repeated implantation failure were included in the study. Thirdly, most of the embryos were transferred at the cleavage stage rather than the blastocyst stage, which limits the potential application of the results. Nevertheless, the current study provided valuable information for clinical practice and research.
In conclusion, the current study demonstrated that HT-FET with 3 cycles of GnRHamay have clinical application for patients with infertility and regular menstrual cycles. However, additional large-scale, double-blinded, randomized controlled trials are required to confirm these results.

\section{Acknowledgements}

The authors wish to thank Miss Lu Dong, Dr Mingxia Gao, Dr Cui Zhao and Mr. Liwei Zhang from The Reproductive Medicine Special Hospital of The First Hospital of Lanzhou University for their assistance with collecting patient data and endometrial biopsies.

\section{Funding}

The current study was funded by the Lanzhou Science and Technology Bureau (grant no. 2017-4-55). Funding supported all molecular experiments.

\section{Availability of data and materials}

The datasets used and/or analyzed during the current study are available from the corresponding author on reasonable request.

\section{Authors' contributions}

LLi and XuZ conceived and designed the study. JA collected endometrial specimens, conducted the study, analyzed data and wrote the manuscript. XiZ collected and partly analyzed the data. LLiu designed the molecular experiments. LW partly collected the data and revised the manuscript. All authors read and approved the final manuscript.

\section{Ethics approval and consent to participate}

Written informed consent was obtained from all participants and the current study was approved by Institutional Ethics Committee of the First Hospital of Lanzhou University (grant no. LDYYLL2018-69).

\section{Patient consent for publication}

Not applicable.

\section{Competing interests}

The authors declare that they have no competing interests.

\section{References}

1. Trounson A and Mohr L: Human pregnancy following cryopreservation, thawing and transfer of an eight-cell embryo. Nature 305: 707-709, 1983.

2. Maheshwari A and Bhattacharya S: Elective frozen replacement cycles for all: Ready for prime time? Hum Reprod 28: 6-9, 2013.

3. McLernon D, Harrild K, Bergh C, Davies MJ, de Neubourg D, Dumoulin JC, Gerris J, Kremer JA, Martikainen H, Mol BW and Norman RJ: Clinical effectiveness of elective single versus double embryo transfer: Meta-Analysis of individual patient data from randomised trials. BMJ 213: 341, 2010. 
4. Veleva Z, Karinen P, Tomas C, Tapanainen JS and Martikainen H: Elective single embryo transfer with cryopreservation improves the outcome and diminishes the costs of IVF/ICSI. Hum Reprod 24: 1632-1639, 2009.

5. Schild RL, Knobloch C, Dorn C, Fimmers R, Van Der Ven H and Hansmann M: Endometrial receptivity in an in vitro fertilization program as assessed by spiral artery blood flow, endometrial thickness, endometrial volume, and uterine artery blood flow. Fertil Steril 75: 361-366, 2001.

6. Groenewoud ER, Cantineau AE, Kollen BJ, Macklon NS and Cohlen BJ: What is the optimal means of preparing the endometrium in frozen-thawed embryo transfer cycles? A systematic review and meta-analysis. Hum Reprod Update 23: 255-261, 2017.

7. Dal Prato L, Borini A, Cattoli M, Bonu MA and Sciajno R: Endometrial preparation for frozen-thawed embryo transfer with or without pretreatment with gonadotropin-releasing hormone agonist. Fertil Steril 77: 956-960, 2002.

8. El-Toukhy T, Taylor A, Khalaf Y, Al-Darazi K, Rowell P, Seed P and Braude P: Pituitary suppression in ultrasound-monitored frozen embryo replacement cycles, A randomized study. Hum Reprod 19: 874-879, 2004.

9. Horne G, Critchlow JD, Newman MC, Edozien L, Matson PL and Lieberman BA: A prospective evaluation of cryopreservation strategies in a two-embryo transfer program. Hum Reprod 12: $542-547,1997$.

10. Queenan JT Jr, Ramey JW, Seltman HJ, Eure L, Veeck LL and Muasher SJ: Transfer of cryopreserved-thawed pre-embryos in a cycle using exogenous steroids without prior gonadotropin-releasing hormone agonist suppression yields favourable pregnancy results. Hum Reprod 12: 1176-1180, 1997.

11. Simon A, Hurwitz A, Zetner BS, Bdolah Y and Laufer N: Transfer of frozenthawed embryos in artificially prepared cycles with and without prior gonadotropin-releasing hormone agonist suppression: A prospective randomized study. Hum Reprod 13: 2712-2717, 1998.

12. Mandelbaum J: Embryo and oocyte cryopreservation. Hum Reprod 15: 43-47, 2000.

13. Hill MJ, Miller KA and Frattarelli JL: A GnRH agonist and exogenous hormone stimulation protocol has a higher live-birth rate than a natural endogenous hormone protocol for frozen-thawed blastocyst-stage embryo transfer cycles: An analysis of 1391 cycles. Fertil Steril 93: 416-422, 2010.

14. Gelbaya TA, Nardo LG, Hunter HR, Fitzgerald CT, Horne G, Pease EE, Brison DR and Lieberman BA: Cryopreserved-thawed embryo transfer in natural or down-regulated hormonally controlled cycles: A retrospective study. Fertil Steril 85: 603-609, 2006.

15. Wang J, Zhou F, Dong Y, Wu R and Qian L: Prolonged gonadotropin-releasing hormone agonist therapy reduced expression of nitric oxide synthase in the endometrium of women with endometriosis and infertility. Fertil Steril 85: 1037-1044, 2006.

16. Liang J, Wang S and Wang Z: Role of microRNAs in embryo implantation. Reprod Biol Endocrinol 15: 90-101, 2017.

17. Kang J, Park J, Chung D, Lee SH, Park EY, Han KH, Choi SJ, Chung IB, Han HD and Jung YS: Comparison of the clinical outcome of frozen-thawed embryo transfer with and without pretreatment with a gonadotropin-releasing hormone agonist. Obstet Gynecol Sci 61: 489-496, 2018.

18. Achache $\mathrm{H}$ and Revel A: Endometrial receptivity markers, the journey to successful embryo implantation. Hum Reprod Update 12: 731-746, 2006.

19. Auernhammer CJ and Melmed S: Leukemia-inhibitory factorneuroimmune modulator of endocrine function. Endocr Rev 21: 313-345, 2000.

20. Bartel DP: MicroRNAs: Genomics, biogenesis, mechanism, and function. Cell 116: 281-297, 2004.

21. Taylor HS: The role of HOX genes in human implantation. Hum Reprod Update 6: 75-79, 2000.

22. Van Mourik MS, Macklon NS and Heijnen CJ: Embryonic implantation: Cytokines, adhesion molecules, and immune cells in establishing an implantation environment. J Leukoc Biol 85: 4-19, 2009.
23. Vuorela P, Carpén O, Tulppala M and Halmesmäki E: VEGF its receptors and the tie receptors in recurrent miscarriage. Mol Hum Reprod 6: 276-282, 2000.

24. Surrey ES, Minjarez DA and Schoolcraft WB: The incidence of aberrant endometrial alphavbeta(3) vitronectin expression in a high risk infertility population: Could prolonged $\mathrm{GnRH}$ agonist therapy play a role? J Assist Reprod Genet 24: 553-556, 2007.

25. Dong X, Sui C, Huang K, Wang L, Hu D, Xiong T, Wang R and Zhang H: MicroRNA-223-3p suppresses leukemia inhibitory factor expression and pinopodes formation during embryo implantation in mice. Am J Transl Res 8: 1155-1163, 2016.

26. Chu B, Zhong L, Dou S, Wang J, Li J, Wang M, Shi Q, Mei Y and Wu M: MiRNA-181 regulates embryo implantation in mice through targeting leukemia inhibitory factor. J Mol Cell Biol 7: 12-22, 2015.

27. Bartel DP: MicroRNAs: Target recognition and regulatory functions. Cell 136: 215-233, 2009.

28. Brosens JJ, Pijnenborg R and Brosens IA: The myometrialjunctional zone spiral arteries in normal and abnormal pregnancies: A review of the literature. Am J Obstet Gynecol 187: 1416-1423, 2002.

29. Gellersen B and Brosens J: Cyclic AMP and progesterone receptor cross-talk in human endometrium: A decidualizing affair. J Endocrinol 178: 357-372, 2003.

30. Paternot G, Debrock S, D'Hooghe T and Spiessens C: Computerassisted embryo selection: A benefit in the evaluation of embryo quality? Reprod Biomed Online 23: 347-354, 2011.

31. Evilsizor MN, Ray-Jones HF, Lifshitz J and Ziebell J: Primer for immunohistochemistry on cryosectioned rat brain tissue: Example staining for microglia and neurons. J Vis Exp 12: e52293, 2015.

32. Itoh H, Mogami H, Bou Nemer L, Word L, Rogers D, Miller R and Word RA: Endometrial stromal cell attachment and matrix homeostasis in abdominal wall endometriomas. Hum Reprod 33: 280-291, 2018

33. Livak KJ and Schmittgen TD: Analysis of relative gene expression data using real-time quantitative PCR and the 2(-Delta Delta C(T)) method. Methods 25: 402-408, 2001.

34. Klemmt PA, Carver JG, Kennedy SH, Koninckx PR and Mardon HJ: Stromal cells from endometriotic lesions and endometrium from womenwith endometriosis have reduced decidualization capacity. Fertil Steril 85: 564-572, 2006.

35. Laird SM, Tuckerman EM and Li TC: Cytokine expression in theendometrium of women with implantation failure and recurrentmis carriage. Reprod Biomed Online 13: 13-23, 2006.

36. Karpovich N, Klemmt P, Hwang JH, McVeigh JE, Heath JK, Barlow DH and Mardon HJ: The production of interleukin-11 and decidualization are compromisedin endometrial stromal cells derived from patients with infertility. J Clin Endocrinol Metab 90: 1607-1612, 2005.

37. Ryan IP and Taylor RN. Endometriosis and infertility: New concepts. Obstet Gynecol Surv 52: 365-371, 1997.

38. Croze F, Kennedy TG, Schroedter IC, Friesen HG and Murphy LJ: Expression of insulin-like growth factor-I and insulin-like growth factor-binding protein-1 in the rat uterus during decidualization. Endocrinology 127: 1995-2000, 1990.

39. Klemmt PA, Liu F, Carver JG, Brosi D, Adamson J, Mardon HJ and McVeigh E: Effects of gonadotrophin releasing hormone analogues on human endometrial stromal cells and embryo invasion in vitro. Hum Reprod 24: 2187-2192, 2009.

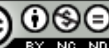

This work is licensed under a Creative Commons Attribution-NonCommercial-NoDerivatives 4.0 International (CC BY-NC-ND 4.0) License. 